\title{
Supporting Information for Projector-Free Capped-Fragment Scheme Within Density Functional Embedding Theory for Covalent and Ionic Compounds
}

John Mark P. Martirez ${ }^{1,2}$ and Emily A. Carter ${ }^{1,2,3^{*}}$

${ }^{1}$ Department of Mechanical and Aerospace Engineering, Princeton University, Princeton, New Jersey, 08544, United States

Current address: ${ }^{2}$ Department of Chemical and Biomolecular Engineering and ${ }^{3}$ Office of the Chancellor, University of California, Los Angeles, Los Angeles, California, 90095, United States

*eac@ucla.edu,eac@princeton.edu 


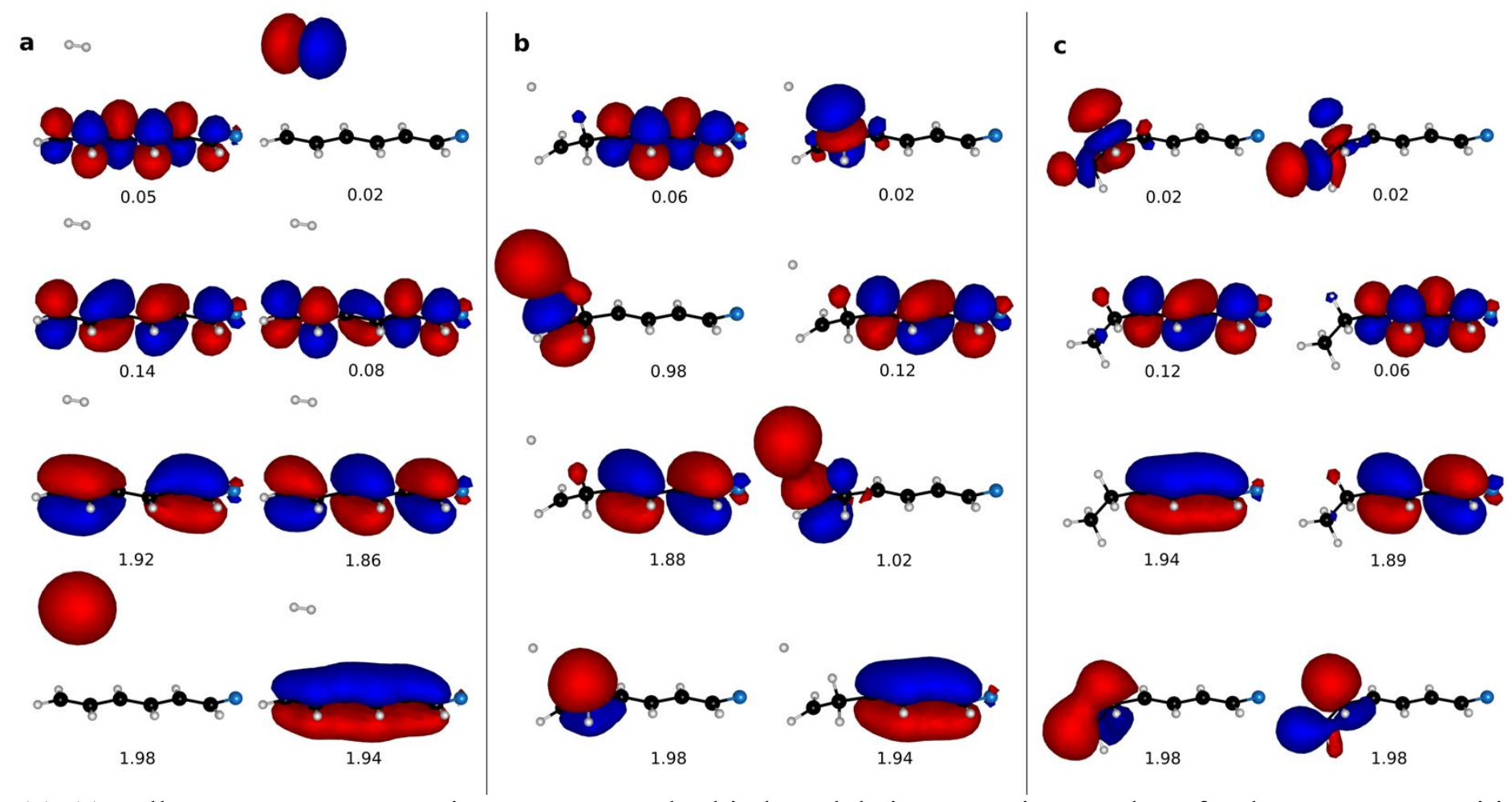

Figure S1. (a)-(c) Full-system CASSCF active-space natural orbitals and their occupation numbers for the reactant, transition state, and product along the MEP for the hydrogenation of 1-fluoro-hexatri-1,3,5-ene $\left(\mathrm{C}_{6} \mathrm{H}_{7} \mathrm{~F}\right)$ described in section 4.1. For the reactant, the $\mathrm{C}$-2pderived three $\pi$ and three $\pi^{*}$ orbitals of $\mathrm{C}_{6} \mathrm{H}_{7} \mathrm{~F}$ (with six electrons) and the $\mathrm{H}-1$ s-derived pair of $\sigma$ and $\sigma^{*}$ orbitals of $\mathrm{H}_{2}$ (with two electrons) define the active space of $(8 \mathrm{e}, 8 \mathrm{o})$. (b)-(c) As the structure evolves into the transition state and product, one and two pairs of $\mathrm{C}-2 \mathrm{p}-\mathrm{H}-1 \mathrm{~s}$-derived $\sigma$ and $\sigma^{*} \mathrm{C}-\mathrm{H}$ orbitals respectively emerge. At the transition state (b), a pair of singly occupied degenerate $\sigma$-like and $\sigma^{*}$-like $\mathrm{C}-\mathrm{H}$ orbitals is present originating from the unsaturated terminal carbon $\left(\mathrm{C}^{6}\right)$ and the unbound $\mathrm{H}$ atom. Isosurface level (blue/red): \pm 0.03 a.u. Black, white, and light-blue spheres: C, H, and F, respectively (see Figs. 2a and 3a). 

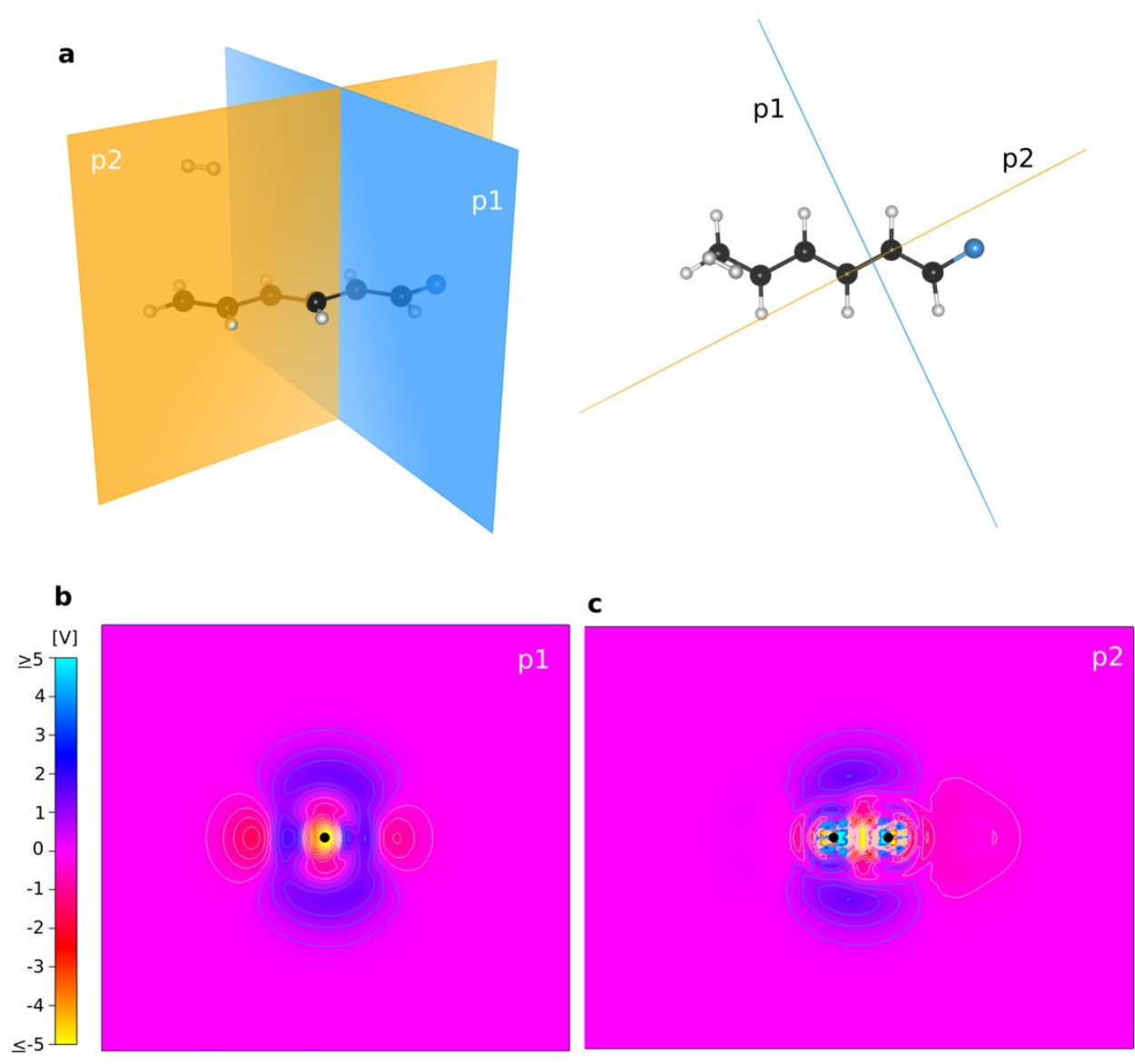

Figure S2. (a) Molecular planes within the $\mathrm{C}_{6} \mathrm{H}_{7} \mathrm{~F}$ molecule at the cleavage point of the fragmentation (between carbon atoms $\mathrm{C}^{2}$ and $\mathrm{C}^{3}$ ). The blue plane (plane 1 or $\mathrm{p} 1$ ) is orthogonal to the cleaved $\mathrm{C}-\mathrm{C}$ bond. The orange plane (p2) contains the cleaved $\mathrm{C}-\mathrm{C}$ bond. Two-dimensional slices of the embedding potential derived for the $\mathrm{C}_{4} \mathrm{H}_{5} \mathrm{H}^{\text {cap }}$ and $\mathrm{C}_{2} \mathrm{H}_{2} \mathrm{FH}^{\text {cap }}$ fragments projected onto planes (b) p1 and (c) p2 shown in (a). The black circles mark the center of the C-C bond in (b) and the positions of $\mathrm{C}^{2}$ and $\mathrm{C}^{3}$ atoms in (c). The maximum and minimum values of the embedding potential are 34.62 and $-48.58 \mathrm{~V}$, respectively, which occur at and near the terminal atoms' nuclei. +5 and $-5 \mathrm{~V}$ upper and lower bounds are chosen in the plots to show the relevant features in the space between and surrounding the terminal atoms. Black, white, and light-blue spheres: C, H, and F, respectively (see Fig. 2a). 

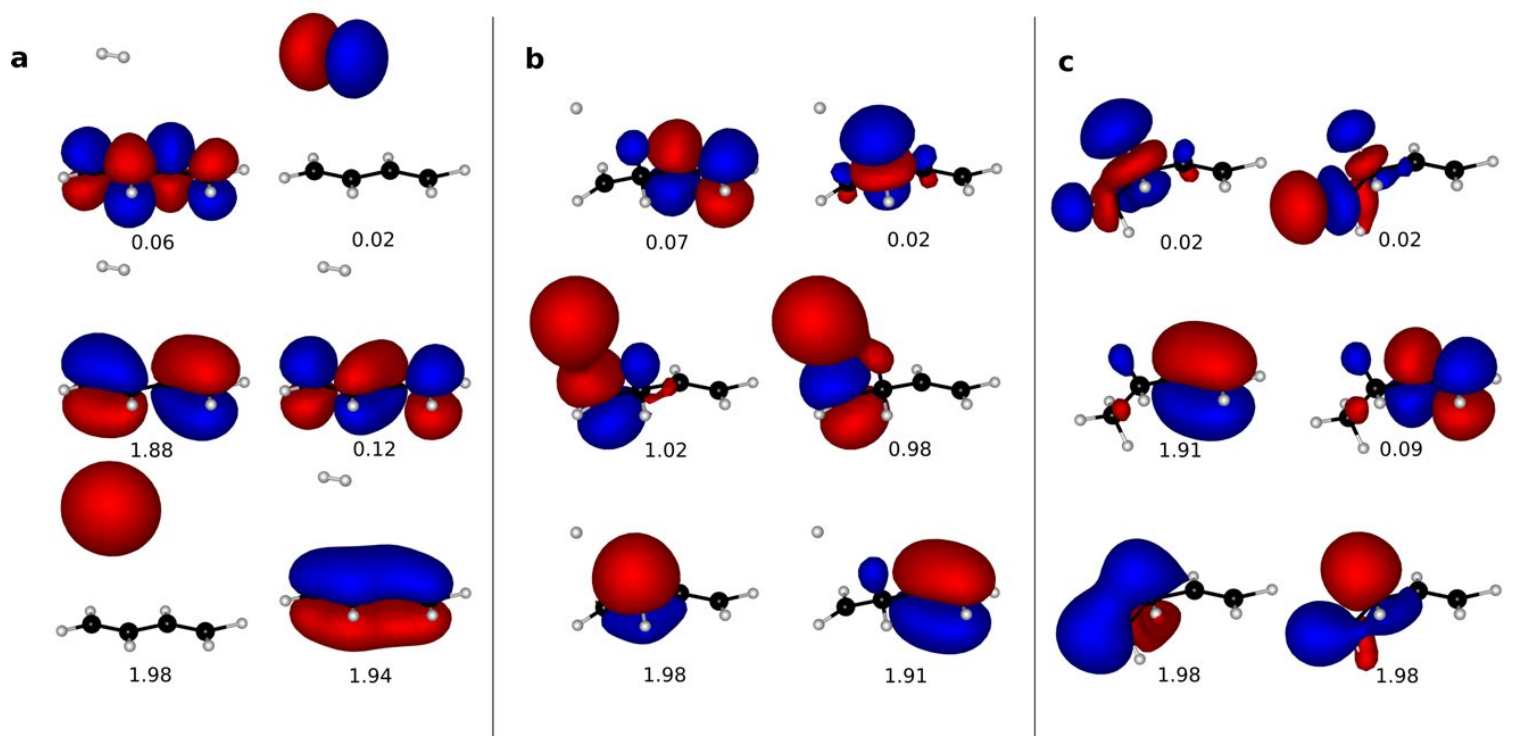

Figure S3. (a)-(c) Embedded- $\mathrm{C}_{4} \mathrm{H}_{5} \mathrm{H}^{\text {cap }}$-fragment $+\mathrm{H}_{2}$ CASSCF active-space natural orbitals and their occupation numbers for the reactant, transition state, and product along the MEP for the hydrogenation of 1-fluoro-hexatri-1,3,5-ene described in section 4.1. (a) Analogous to the fullsystem active space (Fig. S1), for the reactant, the C-2p-derived two $\pi$ and two $\pi^{*}$ orbitals of $\mathrm{C}_{4} \mathrm{H}_{7}$ $\mathrm{H}^{\text {cap }}$ (with four electrons) and the $\mathrm{H}-1$ s-derived pair of $\sigma$ and $\sigma^{*}$ orbitals of $\mathrm{H}_{2}$ (with two electrons) define the active space of (6e, 6o). (b)-(c) Much like in the full-system active-space orbitals (Fig. $\mathrm{S} 1$ ), as the new $\mathrm{C}-\mathrm{H}$ bonds form at the transition state and product, one and two pairs of $\mathrm{C}-2 \mathrm{p}-\mathrm{H}-$ 1s-derived $\sigma$ and $\sigma^{*} \mathrm{C}$-H orbitals respectively form. A pair of singly occupied degenerate $\sigma$-like and $\sigma^{*}$-like $\mathrm{C}$-H orbitals also are present at the transition state (b) - see Fig. S1. Isosurface level (blue/red): \pm 0.03 a.u. Black, white, and light-blue spheres: $\mathrm{C}, \mathrm{H}$, and F, respectively (see Fig. $2 \mathrm{~b}$ ). 

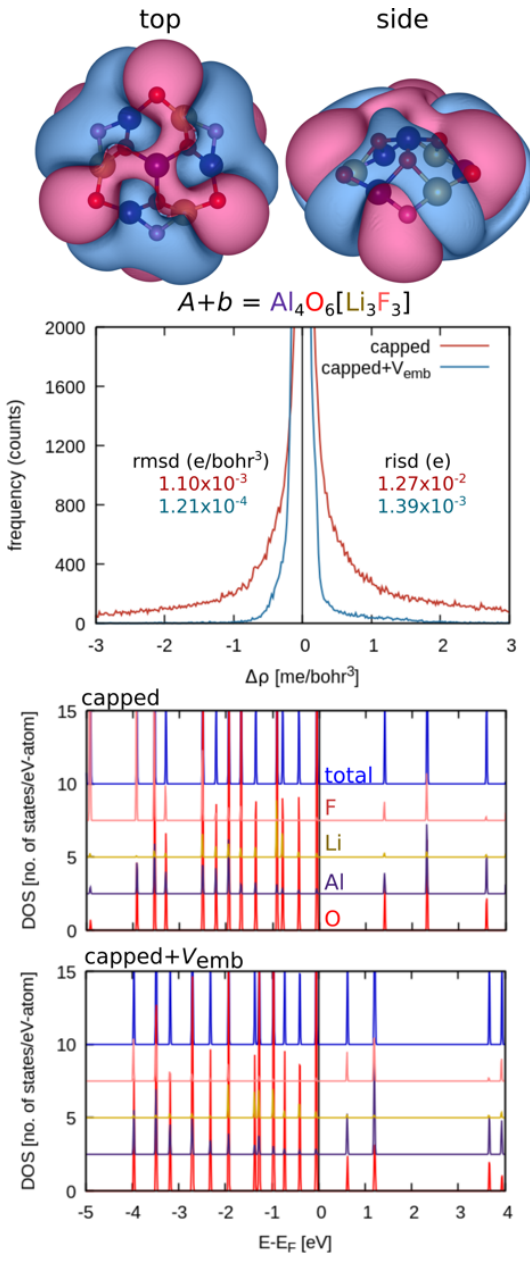

Figure S4. (top) Optimized $V_{e m b}$ in real space showing the repulsive (blue, $+0.1 \mathrm{~V}$ ) and attractive (pink, $-0.1 \mathrm{~V}$ ) potentials for the LiF-capped fragments (Fig. 7f). See also Fig. S5 for the corresponding two-dimensional slices. (middle) Electron density deviation $(\Delta \rho)$ distribution before and after $V_{e m b}$ optimization. (bottom) Projected density of states (DOS) without (top) and with (bottom) the optimized $V_{\text {emb. Note the }}$ metallic character in both LiF-capped and LiF-capped $+V_{\text {emb. }}$. See Fig. 8 in the main text for comparison to cap-free and divalent capping. 


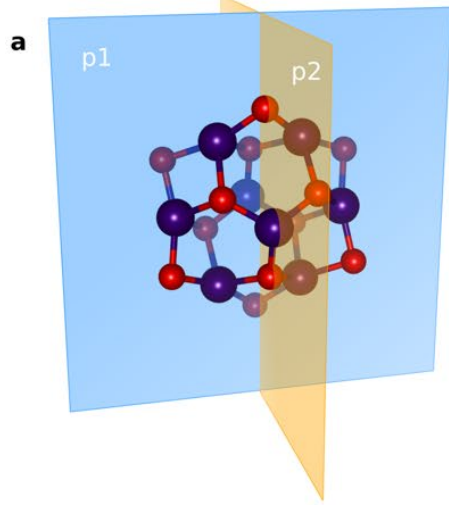

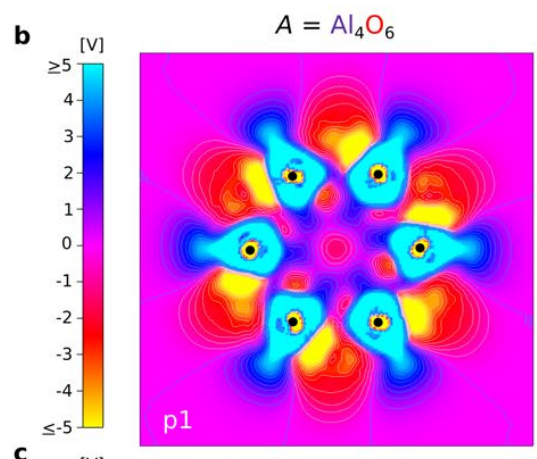

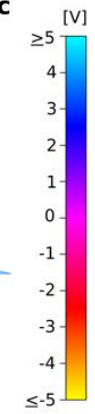

$A+b=\mathrm{Al}_{4} \mathrm{O}_{6}\left[\mathrm{Be}_{3} \mathrm{O}_{3}\right]$

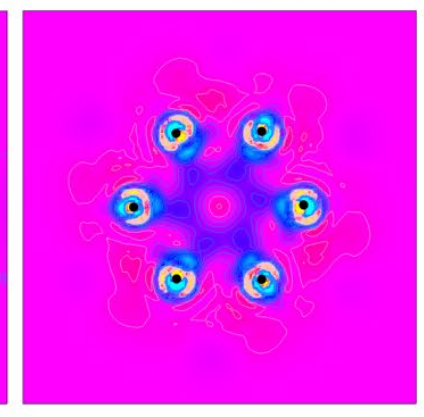

$A+b=\mathrm{Al}_{4} \mathrm{O}_{6}\left[\mathrm{Mg}_{3} \mathrm{O}_{3}\right]$
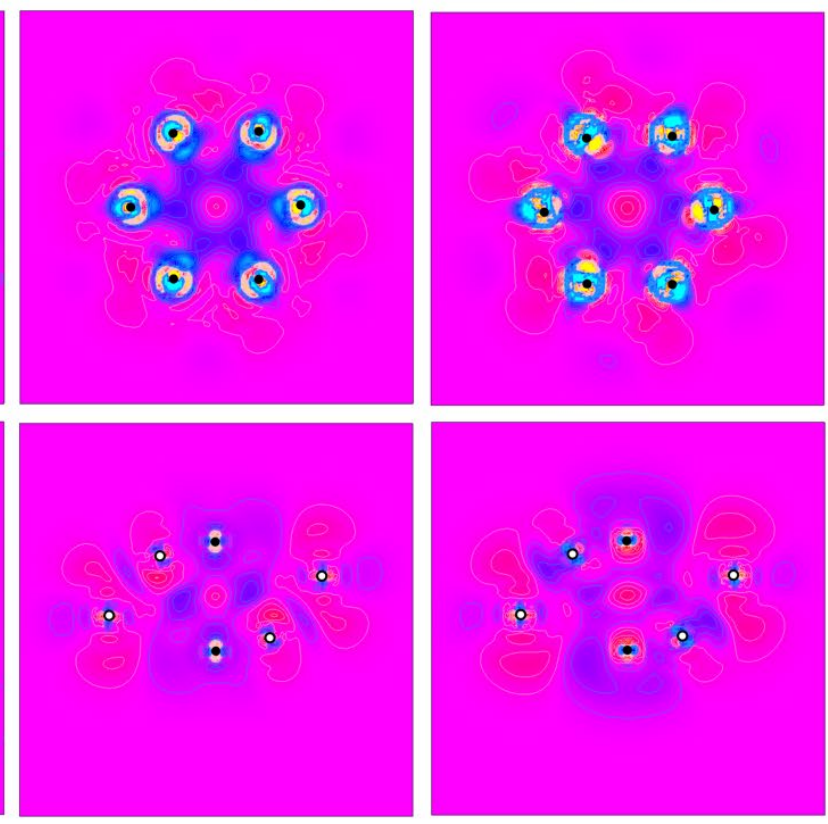

$A+b=\mathrm{Al}_{4} \mathrm{O}_{6}\left[\mathrm{Li}_{3} \mathrm{~F}_{3}\right]$
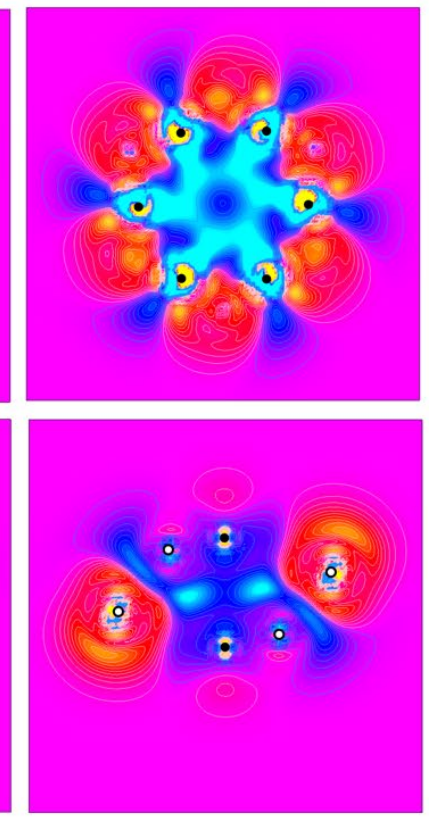

Figure S5. (a) Molecular planes within the $\mathrm{Al}_{8} \mathrm{O}_{12}$ cluster. The blue plane (plane 1 or p1) contains all the terminal Al atoms. The orange plane (p2) is orthogonal to $\mathrm{p} 1$ and contains the two three-fold coordinated Al atoms (see the fragmentation and capping schemes in Figs. 7e and 7f). Two-dimensional slices of the embedding potential derived for the fragments (as labelled) projected onto planes (b) p1 and (c) p2 shown in (a). The black circles mark the positions of the Al nuclei in (b). The black filled and empty circles in (c) mark the positions of the $\mathrm{Al}$ and $\mathrm{O}$ nuclei, respectively. For (b) and (c), the maximum values of the embedding potentials, from left to right, are $29.08,26.71,71.29$, and $65.85 \mathrm{~V}$. The minimum values, from left to right, are $-173.6,-71.49,-41.43$, and $-98.27 \mathrm{~V}$. The extrema occur at and near the terminal atoms' nuclei. +5 and $-5 \mathrm{~V}$ upper and lower bounds are chosen in the plots to show the relevant features in the space between and surrounding the terminal atoms. Violet and red spheres: Al and O, respectively (see Figs. 7a and 7e-f). 